\title{
Cirugía endoscópica en niños y adolescentes con rinosinusitis: Perfil inmunológico y bacteriológico
}

\author{
Endoscopic sinus surgery in children and adolescent with rhino sinusitis: \\ Immunologic and bacteriologic profile
}

\author{
María José Herrera $\mathrm{J}^{1,2}$, Gloria Ribalta $\mathrm{L}^{3}$.
}

\begin{abstract}
RESUMEN
Introducción: La rinosinusitis, corresponde a un grupo de desórdenes que se caracteriza por la inflamación de la mucosa tanto de cavidades paranasales (CPN) como de fosas nasales. Está determinada por factores del huésped y ambientales que interactúan causando un estado inflamatorio persistente que produce remodelación de la mucosa. Entre éstos se destacan la alergia y otras alteraciones inmunológicas junto con la colonización por agentes infecciosos.

Objetivo: Determinar las características demográficas, clínicas, inmunológicas y agentes infecciosos involucrados en la rinosinusitis crónica ( $R S C$ ) y rinosinusitis aguda ( $R S A$ ) recurrente de niños y adolescentes sometidos a cirugía endoscópica funcional (CEF). Establecer las posibles asociaciones de estas características con la necesidad de reoperación y la recurrencia de la enfermedad.

Material y método: Estudio retrospectivo observacional en que se evalúan 28 niños y adolescentes sometidos a CEF en el servicio de ORL de Clínica Las Condes durante los años 2000 a 2009. Todos los pacientes contaban con estudio inmunológico y test cutáneo preoperatorio más biopsia de mucosa de CPN y cultivos intraoperatorios de secreción de CPN. Los análisis estadísticos se realizaron con test de Anova para los análisis descriptivos y para el análisis de grupos el test de Fisher. Considerando como significativo un $p<0,05$.

Resultados: Se analizaron 28 pacientes; el 89,2\% tuvo test cutáneo positivo y biopsia inflamatoria crónica eosinofílica (53,6\%). El 17\% presentó algún tipo de deficiencia inmunológica. Se obtuvo cultivos positivos intraoperatorios en el $71 \%$ de las muestras, en su mayoría agentes aeróbicos (57,1\%), con 14,2\% de anaerobios y sin presencia de hongos.

Veinte y siete pacientes tuvieron seguimiento posoperatorio de 5 años. Cuatro de ellos (14\%) debió ser reoperado. Durante el periodo de seguimiento el $22 \%$ desarrolló recurrencia de rinosinusitis aguda. Se consideró una mala evolución posquirúrgica la necesidad de reoperación o la recurrencia de $R S A$. No se logró encontrar asociación de las variables estudiadas con una mala evolución posquirúrgica.

Conclusión: No se logró demostrar una asociación entre los factores que interactúan en la génesis de la RSC y $R S A$ recurrente y su mala evolución posoperatoria en esta serie estudiada. Sin embargo, podemos tener una orientación de cómo es el estado inmune y bacteriológico de los niños sometidos a cirugía en nuestro medio.
\end{abstract}

Palabras clave: Rinosinusitis, niños, cirugía endoscópica funcional, recurrencia.

\footnotetext{
Médico Becado Otorrinolaringología, Hospital Barros Luco Trudeau.

Magíster en Ciencias Médicas mención en Inmunología, Universidad de Chile.

Médico Otorrinolaringólogo, Servicio Otorrinolaringología, Clínica Las Condes.
} 


\section{ABSTRACT}

Introduction: Rhino sinusitis is a group of disorders characterized by inflammation of the mucosa of both sinuses and nostrils. It is determined by environmental and host factors that interact causing a persistent inflammatory condition that causes remodeling of the mucosa. Chief among these are allergy and other immune disorders with colonization by infectious agents.

Aim: To determine the demographic, clinical, immunologic and infectious agents involved in chronic Rhino sinusitis (CRS) and Recurrent Acute Rhino sinusitis (RAR) in children and adolescents undergoing functional endoscopic sinus surgery (FESS). Establish the possible associations of these characteristics with the need for re operation and recurrence of the disease.

Material and method: Retrospective observational study that evaluated 28 children and adolescents involved in the ENT department at Clínica Las Condes, Santiago, Chile during the years 2000 to 2009. All study patients had preoperative Prick test, immune workout and intraoperative Para nasal mucosal biopsy and cultures of Para nasal secretion. Statistical analysis was performed using ANOVA test for descriptive analysis and cluster analysis with Fisher Exact test. Results were considered significant with $p<0.05$

Results: We analyzed 28 patients, $89.2 \%$ had positive Prick test and $53.6 \%$ showed biopsy with eosinophilic inflammatory disease. $17 \%$ had some type of immune deficiency. Positive intraoperative cultures were obtained in $71 \%$ of the samples, mostly aerobic agents (57.1\%), with $14.2 \%$ anaerobes and negatives cultures for fungi.

Twenty seven patients had postoperative follow-up of 5 years. Four of them (14\%) had to be re-operated. During the monitoring period, $22 \%$ developed RAR. It was considered a poor surgical outcome the need for re-operation or recurrence of RAR. We couldn't find association of variables with a poor outcome after surgery.

Conclusion: It was not possible to demonstrate an association between the interacting factors in the genesis of CRS and RAR and poor surgical outcome in the studied series. However, we do have an orientation of how is the immune status and bacteriology of children undergoing surgery in our midst.

Key words: Rhino sinusitis, children, functional endoscopic sinus surgery, recurrence.

\section{INTRODUCCIÓN}

La rinosinusitis (RS) se ha definido como un grupo de desórdenes caracterizado por la inflamación tanto de la mucosa de los senos paranasales como de las fosas nasales ${ }^{1}$. Se clasifica en aguda o crónica según la duración de los síntomas, considerándose RSC cuando dura 12 semanas 0 más ${ }^{2,3}$. La RSC puede clasificarse según la presencia concomitante de pólipos nasales. Asociándose a una respuesta de tipo Th2 cuando éstos están presentes y de tipo Th1 cuando no lo están ${ }^{3,4}$. También se describe a la RS alérgica fúngica (AFRS).

La RSC posee una gran variabilidad en su presentación clínica, ya que está determinada por múltiples factores del huésped y del ambiente. La Aca- demia Americana de Otorrinolaringología y Cirugía de Cabeza y Cuello (AAO) elaboró una lista de síntomas y signos clínicos (hechos) para realizar el diagnóstico de RS, considerando necesaria la presencia de al menos 2 factores mayores, 0 uno mayor y 2 menores. Los hechos mayores son dolor y/o presión facial, congestión facial, obstrucción nasal, rinorrea purulenta, hiposmia, anosmia y hallazgo de pus al examen. Cefalea, decaimiento, dolor dentario, halitosis, tos y otalgia son hechos menores ${ }^{1}$.

El tratamiento médico de esta patología consiste en el uso de antibióticos y corticoides nasales, junto con el manejo de los factores inflamatorios asociados (alergia respiratoria, intolerancia a la aspirina, reflujo gastroesofágico, etc.). La cirugía endoscópica nasosinusal (CEF) sólo se reserva para 
aquellos casos en que el tratamiento médico máximo no logre resultados satisfactorios. En niños este punto cobra mayor relevancia puesto que la cronicidad suele ser reversible con un adecuado tratamiento médico en un mayor número de casos comparado con los adultos. Es por esto que el porcentaje de niños que llega a CEF cada día es menor si uno analiza su propia experiencia y la de la literatura publicada del tema ${ }^{5,6}$.

Un pequeño porcentaje puede asociarse a enfermedades genéticas como la fibrosis quística (FQ). En estos pacientes los defectos en el clearance mucociliar, que también se observan en la disquinesia ciliar primaria (DCP), aumentan el riesgo de desarrollar la enfermedad².

En la etiología de la RSC múltiples factores ambientales y del huésped pueden estar actuando de manera simultánea o independiente. Entre éstos se ha descrito:

a. Alergia y otras alteraciones inmunológicas: En diversos estudios se encuentra una asociación entre alergia y RSC, entre el $25 \%$ y $75 \%{ }^{5}$. La alergia mediada por IgE está en el $60 \%$ de los pacientes con RSC, principalmente a alergenos perennes. También se observa sensibilidad a hongos en pacientes con AFRS, cuadro poco frecuente en niños ${ }^{8}$. Estudios histopatológicos en pacientes con RSC han mostrado infiltración linfocitaria y citoquinas de tipo $\mathrm{Th}^{9}$.

b. Se ha encontrado baja producción de anticuerpos o hipogamaglobulinemia en $12 \%$ de RSC sin pólipos. No se ha descrito asociación con otras inmunodeficiencias ${ }^{10}$.

c. Colonización de mucosa nasosinusal: El Staphylococcus aureus secreta toxinas (superantígenos bacterianos) que estimulan potentemente linfocitos $T$, liberación de citoquinas y una respuesta policlonal de lgE, 10 que induce reclutamiento de eosinófilos produciendo cambios histopatológicos y clínicos asociados a RSC con pólipos ${ }^{3}$.

d. Los biofilms son agregados bacterianos que forman una red de microorganismos cubiertos por una película de glicocálix. Esta matriz extracelular protectora, les otorga resistencia antimicrobiana y protección contra el huésped y el ambiente permitiendo su multiplicación. Tienen un rol indiscutible en el desarrollo de RSC y su mala respuesta a tratamiento médico. Se están desarrollando nuevas terapias médicas, pero por ahora parecen erradicables sólo mediante cirugía ${ }^{12,13}$. En niños, la presencia de biofilms en adenoides ha sido demostrado ya en varios trabajos ${ }^{11}$. Es por esto que se considera la adenoidectomía como la primera intervención quirúrgica en los casos de niños con RSC o RSA recurrente sin respuesta a tratamiento médico máximo.

e. La colonización por hongos induce y mantiene la inflamación mediada por eosinófilos y linfocitos nasales, pero no es claro su rol patogénico3.

f. Cuando se evalúa la presencia de eosinófilos en biopsias de RSC se observan resultados contradictorios. Baroody y cols compararon el número de eosinófilos en tejidos de niños con RSC en relación a mucosa normal de seno esfenoidal de adultos sin historia RS. Todos los niños tuvieron un mucho mayor número de eosinófilos que los normales. Los eosinófilos estarían presentes en la mucosa inflamada independiente del estado alérgico ${ }^{14}$. Sin embargo, Chan6, encuentra una menor proporción de eosinófilos en la submucosa de pacientes pediátricos con RSC antes de los 8 años, estableciendo la hipótesis que antes de esa edad, el factor de cronicidad en niños sería reversible.

g. Reflujo gastroesofágico (RGE): La RS se ha considerado como una manifestación extradigestiva del RGE. Se cree que el reflujo de ácido gástrico a faringe y nasofaringe, produce inflamación del ostium sinusal, derivando en sinusitis ${ }^{15,17}$. Estudios prospectivos demuestran que los niños con RSC presentan más episodios de reflujo que la población general, pero no son concluyentes. Por otra parte, el tratamiento con inhibidores de la bomba de protones (PPI) disminuye el número de pacientes pediátricos con RSC que tienen indicación quirúrgica ${ }^{18}$.

Múltiples antecedentes muestran que el desarrollo de la RSC depende de la interacción entre factores de susceptibilidad del huésped y factores ambientales. Estos se caracterizan por determinar un estado inflamatorio crónico y persistente que produce remodelación de la mucosa nasosinusal ${ }^{19,20}$. Basados en esta teoría es que se realiza una evalua- 
ción de los factores participantes en la fisiopatología de la enfermedad en niños con RSC y RSA recurrente.

El objetivo de este trabajo fue determinar las características demográficas, clínicas, inmunológicas y de los agentes colonizadores en niños sometidos a CEF por RSC y RSA recurrente, e intentar establecer asociaciones de estos factores con la recurrencia de la enfermedad.

\section{MATERIAL Y MÉTODO}

Se realiza un estudio retrospectivo observacional en que se evalúan los datos clínicos de niños y adolescentes sometidos a CEF durante Ios años 2000 a 2009, por el autor senior, en el Departamento de Otorrinolaringología de Clínica Las Condes, Santiago, Chile. Los criterios de inclusión fueron: pacientes menores de 18 años, portadores de RSC o RSA recurrente según los criterios de la $A A 0^{1}$. Todos los pacientes debían tener tomografía computarizada de cavidades perinasales (TC CPN) con cambios inflamatorios cuantificados por la escala de Lund Mac-Kay (no reversibles luego de tratamiento médico máximo y/o adenoidectomía), biopsia posoperatoria de la mucosa de CPN y estudio inmunológico preoperatorio, que incluyó test cutáneo a alérgenos inhalatorios y alimentarios, niveles plasmáticos de inmunoglobulinas $\mathrm{G}, \mathrm{M}, \mathrm{A}$ y $\mathrm{E}$, subclases IgG, título de anticuerpos antineumococo posvacuna y subpoblación linfocitaria. Como criterio de exclusión se tomó la CEF por complicación de RSA.

Los análisis estadísticos se realizaron con test de Anova para los análisis descriptivos y para el análisis de grupos el test de Fisher. Considerando como significativo un $p<0,05$.

\section{RESULTADOS}

Pacientes: Durante el periodo analizado se obtuvo un total de 35 pacientes que cumplían con los criterios de inclusión. Las características de la muestra eran: edad promedio de cirugía $11 \pm 4$ años, con un rango de 3 a 17 años. Mujeres $48,6 \%$, hombres $51,4 \%$.

El diagnóstico preoperatorio fue RSA recurrente $14,3 \%$, RSC sin pólipos $20 \%$, RSC con pólipos
5,5\%, síndrome de seno silencioso $14,3 \%$, mucocele $2,9 \%$, RSC asociado a DCP 22,9\%, RSC con FQ $17,1 \%$, RSC con DCP y FQ 2,9\%. Dada la diferente característica del cuadro de RSC que se presenta en Ios pacientes con FQ, se decidió eliminar del grupo de estudio a estos pacientes, reduciéndose nuestro universo de estudio a 28 pacientes.

La mayoría de los pacientes fueron intervenidos con CEF bilateral y se trataba de adolescentes con mayor número de cavidades perinasales comprometidas como se puede ver en la Tabla 1 , en base al compromiso de éstas en el TC CPN según puntaje de Lund-Mackay. A sólo 6 de los 28 pacientes se les realizó en conjunto con la CEF adenoidectomía, puesto que el resto del grupo habían sido adenoidectomizados previamente como primer tratamiento quirúrgico de la RSA recurrente y RSC en el niño, sin resultados satisfactorios, por lo que se debió recurrir a la CEF. En los casos de niños menores, con mayor puntaje de Lund-MacKay, se efectuó miniCEF consistente en: uncinectomía, plastía del ostium del seno maxilar y apertura de bula etmoidal según la técnica descrita por Lusk ${ }^{16}$.

Los casos en que sólo se hizo CEF de senos maxilares corresponden a síndrome de seno maxilar silencioso en los que se hizo uncinectomía y plastía del ostium del seno maxilar comprometido.

El tipo de cirugía realizado se resume en la Figura 1. La relación del tipo de cirugía y el puntaje de Lund- Mackay se muestra en la Tabla 1.

El estudio inmunológico mostró que el 89,2\% de los pacientes tenían test cutáneo positivo, encontrándose una mayor presencia de componente alérgico en estos pacientes, en relación a la población general, ver Figura 2. La mayor parte, 53,6\%, presentaba biopsia inflamatoria crónica eosinofílica. La asociación no fue estadísticamente significativa, lo que se muestra en la Figura 3. Del total de pacientes sólo $17,9 \%$ presentaron deficiencia inmunológica, de los cuales 3 tenían déficit de anticuerpo antineumoniae, 1 deficiencia de Natural Killer y 1 hipogammaglobulinemia.

Los cultivos obtenidos de secreción purulenta intrasinusal fueron positivos en el $71 \%$ de los casos, en su mayoría gérmenes aeróbicos. No se obtuvo cultivos positivos para hongos, como se describe en la Figura 4. Los agentes encontrados se presentan en la Figuras 5 y 6 . Se encontró más de 1 germen en 5 pacientes, los cuales tuvieron en cada 


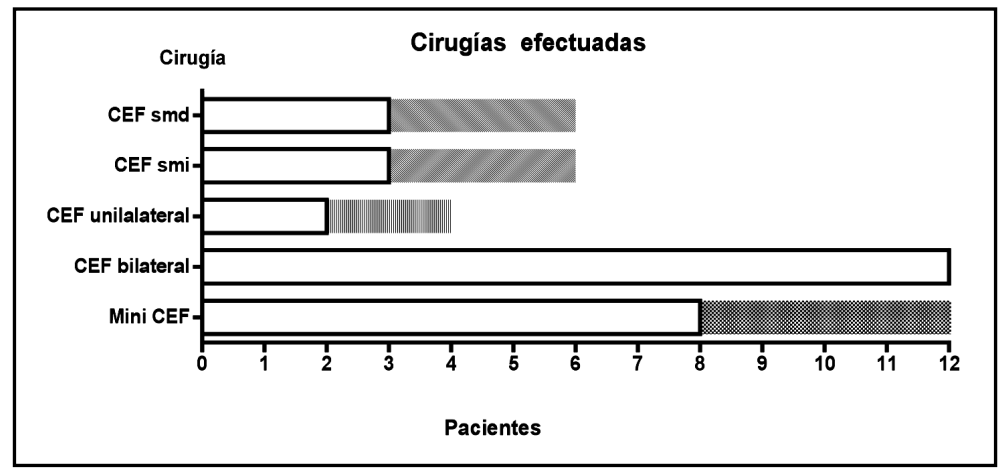

Figura 1. Cirugías efectuadas. Se muestran los tipos de CEF realizadas. CEF smd: cirugía endoscópica funcional de seno maxilar derecho, CEF smi; cirugía endoscópica funcional de seno maxilar izquierdo, CEF unilateral: cirugía endoscópica funcional unilateral, CEF bilateral: cirugía endoscópica bilateral, Mini CEF: uncinectomía, plastía del ostium de seno maxilar y apertura de bula etmoidal.

Tabla 1. Relación tipo de operación y puntaje Lund-Mackay. (Grado de compromiso de CPN en TC CPN)

\begin{tabular}{|lc|}
\hline Operación & Puntaje Lund-Mackay \\
\hline Mini CEF & 12,9 \\
CEF smi o smd & 3,3 \\
CEF unilateral & 7 \\
CEF bilateral & 16,08 \\
\hline
\end{tabular}

Se destaca el hecho que a mayor número de cavidades perinasales comprometidas más extensa el tipo de cirugía. La mini CEF fue realizada en niños menores y la CEF bilateral en adolescentes.

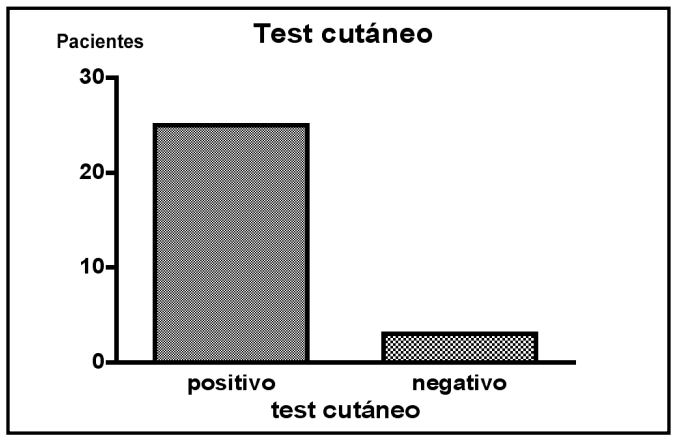

Figura 2. Estudio inmunológico. El test cutáneo fue positivo en el $89,2 \%$ del grupo estudiado.

caso, $S$ aureus y $H$ influenzae, $H$ influenzae y stenotrofomona, $M$ catarrallis y $H$ influenzae, $P$ bacterium y $S$ coagulasa negativo, peptostreptococo y $S$ coagulasa negativo.

De los 28 pacientes analizados, 27 pacientes fueron seguidos por un período promedio de 5 años

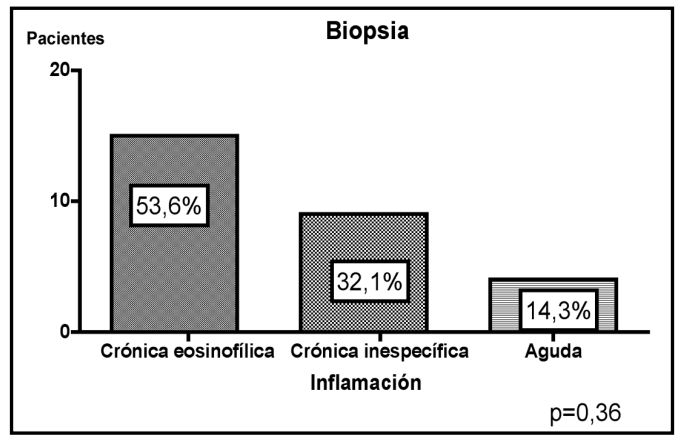

Figura 3. Resultados de las biopsias posoperatorias; el 53,6\% presentó inflamación crónica con infiltrado eosinofílico.

y 2 meses, 1 paciente se cambió de ciudad y no tuvo seguimiento. Sólo 4 de ellos fueron sometidos a reoperación, lo cual se muestra en la Figura 7 . Las reoperaciones se realizaron en la mitad de los pacientes por sinequia de cornete medio, y la otra mitad por recidiva de RSC por DCP. Luego del segui- 


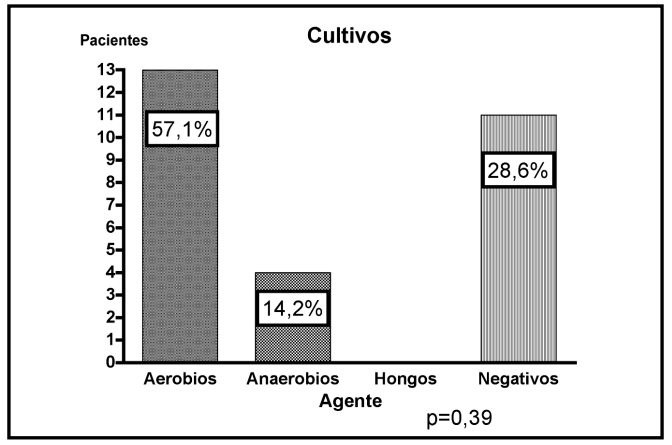

Figura 4. Cultivos. Resultado de los cultivos intraoperatorios.

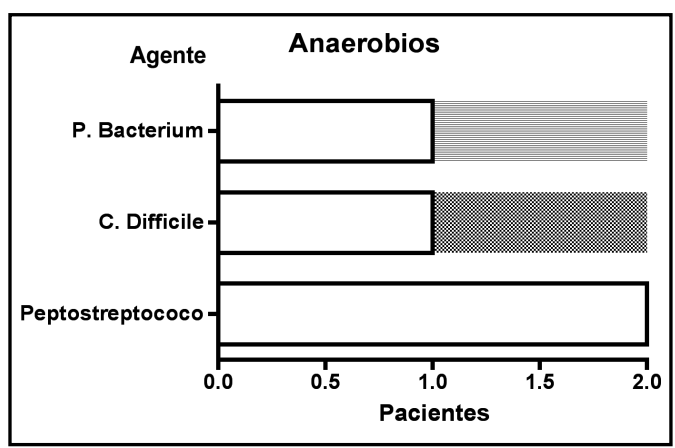

Figura 6. Tipos de agentes patógenos. Los agentes anaerobios encontrados en cultivos intraoperatorios.

miento $78 \%$ no tuvo RS, y el $22 \%$ desarrolló al menos un episodio de RSA.

Con el objetivo de determinar si existe alguna condición que represente un factor de riesgo en el pronóstico de estos pacientes, se realizó análisis de los pacientes de acuerdo a su estado en su último control, considerando como no fracaso aquellos que se mantuvieron sanos, y como fracaso los que desarrollaron RSA o se reoperaron. No se encontró asociación estadísticamente significativa entre los 2 grupos para ninguna variable. Tabla 2.

\section{DISCUSIÓN}

Este trabajo nos permite tener una orientación de cómo es el estado inmune y bacteriológico de los niños con RSC sometidos a cirugía en nuestro medio.

La población estudiada son sólo los pacientes operados, no tenemos universo de todos los que

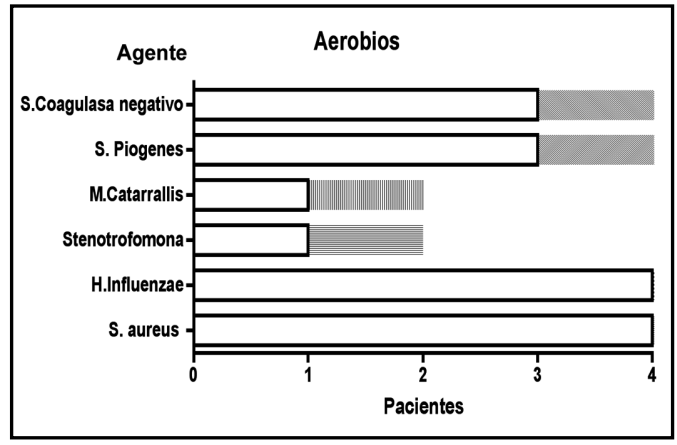

Figura 5. Tipos de agentes patógenos. Los agentes aerobios encontrados en cultivos intraoperatorios.

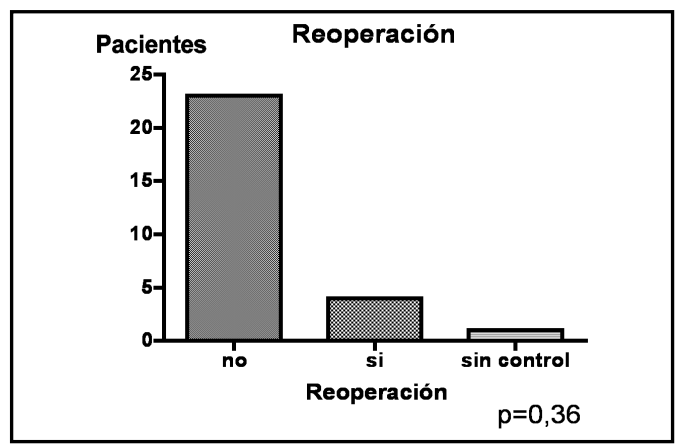

Figura 7. Reoperación. Cuatro reoperaciones realizadas en un período de seguimiento de 5 años y 2 meses; 2 por sinequia posoperatoria en cornete medio y 2 por recidiva de RSC en pacientes con DCP.

consultan por RSA recurrente o RSC, por lo que el análisis no considera pacientes que resuelven su cuadro sólo con tratamiento médico.

La mayor parte de estos niños presentaban componente atópico, en este grupo la mayoría tenía biopsia con infiltrado eosinofílico inflamatorio crónico, compatible con rinitis alérgica $(\mathrm{RA})$. Consideramos que en la niñez, la presencia de RA en el desarrollo de RSC es de gran importancia. En menores el factor crónico irreversible, como alteraciones anatómicas, sería menos frecuente. Fortaleciendo este concepto, en un estudio prospectivo de niños con RA mostró una prevalencia de $53 \%$ de radiografías de CPN alteradas. Lo mismo se observó, al evaluar niños atópicos, con $70 \%$ con anormalidad en radiografía de CPN²1.

Debido a que la patología alérgica se resuelve con tratamiento médico, hay que extremar los esfuerzos en esta área antes de considerar la cirugía. Sin embargo, siempre debemos buscar factores contri- 
Tabla 2. Análisis por grupos

\begin{tabular}{|lccc|}
\hline Variable & $\begin{array}{c}\text { Grupo no fracaso } \\
\mathrm{n}=19\end{array}$ & $\begin{array}{c}\text { Grupo fracaso } \\
\mathrm{n}=9\end{array}$ & Valor de $\mathrm{p}$ \\
\hline Test cutáneo positivo & $89,4 \%$ & $88,8 \%$ & 0,7 \\
Biopsia crónica eosinófilica & $52,6 \%$ & $55,5 \%$ & 0,6 \\
Biopsia crónica inespecífica & $36,80 \%$ & $22,20 \%$ & 0,67 \\
Biopsia inflamatoria aguda & $10,50 \%$ & $22,20 \%$ & 0,57 \\
Cultivos aerobios & $42,10 \%$ & $55,50 \%$ & 0,58 \\
Cultivos anaerobios & $10,50 \%$ & $22,20 \%$ & 0,57 \\
\hline
\end{tabular}

Análisis de pacientes de acuerdo a su estado en último control. No fracaso: sanos. Fracaso: desarrollo de RSA o reoperación.

buyentes que lleven a cronicidad y estar atentos a la indicación quirúrgica oportuna cuando se tema que el caso se está tornando irreversible. Esto último cobra mayor importancia en el caso del síndrome de seno maxilar silencioso, del cual tuvimos 2 casos y donde la intervención a tiempo evita la atelectasia del seno y sus probables consecuencias futuras (descenso del globo ocular, diplopía, asimetría facial).

En cuanto a las deficiencias inmunológicas, se encontró $17,9 \%$ de pacientes con este tipo de alteraciones. Este porcentaje de alteraciones resulta mayor que el presente en la población general donde la incidencia de inmunodeficiencias primarias es de 1 en 10.000 recién nacidos vivos ${ }^{22}$.

No se estudió RGE, el cual es otro factor de riesgo importante de esta patología en niños. En un estudio caso control realizado en niños con RGE se encontró $4,2 \%$ de asociación con RSC versus $1,4 \%$ en el grupo control $(p<0,0001)^{23}$. Otro estudio en niños con RSC realizó pHmetría de 24 hrs con sensor en nasofaringe y esófago distal, observándose $63 \%$ de RGE versus $5 \%$ en la población general. El $79 \%$ reportó mejoría de los síntomas de RSC con tratamiento médico del RGE24.

En nuestro estudio los cultivos intraoperatorios muestran bajo porcentaje de anaerobios (14,2\%), pese a que las muestras fueron enviadas a cultivo de inmediato y en tubos de hemocultivos, para optimizar el crecimiento de este tipo de gérmenes. Otros autores han realizado cultivos en pacientes con RSC que fallaron al tratamiento médico agresivo, encontrándose patógenos aerobios como Streptococcus alpha-hemolitico, Staphylococcus aureus, Moraxella catarrhalis, Streptococcus pneumoniaey Haemophilus influenzaey anaerobios en 6\%. La mayoría de los estudios realizados son de cultivos intraoperatorios de CEF25 al igual que los nuestros. Este tipo de cultivos tiene el sesgo del uso de antibióticos preoperatorios como parte del tratamiento médico máximo, lo que resulta en gérmenes seleccionados y altera la verdadera flora microbiana de la RSC y RSA recurrente sin mediar el uso de antimicrobianos.

Estudios muestran una mayor severidad del cuadro, tanto clínicamente como en el TC CPN, en pacientes con RA. En nuestra muestra, luego del seguimiento posoperatorio, el $22 \%$ desarrolló RSA y 4 tuvieron que ser reoperados. De estos pacientes la mayoría tenía una biopsia con infiltrado inflamatorio eosinófilo. No se observó asociación entre el estado inmunológico de los pacientes, ni la inflamación local, o los agentes patógenos con la recurrencia de la enfermedad. Es probable que el tamaño de la muestra no permita establecer asociaciones.

Los análisis estadísticos no muestran diferencias estadísticamente significativas, por lo que se deben considerar factores como el bajo número de pacientes, error en la técnica, y la selección de la flora por el tratamiento antibiótico previo.

\section{CONCLUSIÓN}

No logramos demostrar asociación entre los factores que interactúan en esta patología y su mala evolución. Sin embargo, al menos en este grupo de pacientes, pudimos observar mayor positividad de test cutáneo, presencia de eosinofília en la biopsia de mucosa de las CPN involucradas en la RSC o RSA recurrente y un porcentaje no despreciable de gérmenes anaerobios en las secreciones intrasinusales al momento de la CEF. 


\section{BIBLIOGRAFÍA}

1. Meltzer e0, Hamilos Dl, Hadley ja, et al. Rhinosinusitis: Establishing definitions for clinical research and patient care. Otolaryngol Head Neck Surg 2004; 131(6 Suppl): S1-62.

2. Dykewicz MS, Hamilos DL. Rhinitis and sinusitis. The Journal of Allergy and Clinical Immunology 2011; 125(2 Suppl 2): S103-15.

3. Kern RC, Conley DB, Walsh W, et al. Perspectives on the etiology of chronic rhinosinusitis: an immune barrier hypothesis. American Journal of Rhinology 2008; 22(6): 549-59.

4. Van Zele T, Claeys S, Gevaert P, et al. Differentiation of chronic sinus diseases by measurement of inflammatory mediators. Allergy 2006; 61(11): 1280-9.

5. Ahmad N, Zacharek MA. Allergic rhinitis and rhinosinusitis. Otolaryngologic Clinics of North America 2008; 41(2): 267-81, v.

6. Chan K, Abzug M, Coffinet L, Simoes E, Cool D, Liu A. Chronic rhinosinusitis in young children differs from adults: a histopathology study. Journal of Pediatrics 2004; 144 ( 2): 206-12.

7. Baroody FM, Hughes CA, McDowell P, Hruban $R$, ZINREICH SJ, NACLERIO RM. Eosinophilia in chronic childhood sinusitis. Archives of OtolaryngologyHead \& Neck Surgery 1995; 121(12): 1396-402.

8. Berrettini S, Carabelli A, Sellari-Franceschini S, ET AL. Perennial allergic rhinitis and chronic sinusitis: correlation with rhinologic risk factors. Allergy 1999; 54(3): 242-8.

9. Hamilos DL, Leung DY, Wood R, et al. Evidence for distinct cytokine expression in allergic versus nonallergic chronic sinusitis. The Journal of Allergy and Clinical Immunology 1995; 96(4): 537-44.

10. Carr TF, KoterbaAP, Chandra R, et al. Characterization of specific antibody deficiency in adults with medically refractory chronic rhinosinusitis. American Journal of Rhinology \& Allergy 2011; 25(4): 241-4.

11. Zuliani G, Carron M, Gurrola J, et al. Identification of adenoid biofilms in chronic rhinosinusitis. Int $J$ Pediatr Otorhinolaryngol 2006; 70(9): 1613-7.

12. Suh JD, Cohen NA, Palmer JN. Biofilms in chronic rhinosinusitis. Current Opinion in Otolaryngology \& Head and Neck Surgery 2010; 18(1): 27-31.
13. Hai PV, Lidstone C, Wallwork B. The effect of endoscopic sinus surgery on bacterial biofilms in chronic rhinosinusitis. Otolaryngol Head Neck Surg 2011; 142(3 Suppl 1): S27-32.

14. BARBERo GJ. Gastroesophageal reflux and upper airway disease. Otolaryngologic Clinics of North America 1996; 29(1): 27-38.

16. Lusk R. Pediatric chronic sinusitis in: Flint: Cummings Otolaryngology: Head \& Neck Surgery, 5th ed. Copyright(c) 2010 Mosby, chapter 19: 277-8.

17. Schrelier S, Garten D, SudHoff H. Pathophysiological mechanisms of extraesophageal reflux in otolaryngeal disorders. Eur Arch Otorhinolaryngol 2009; 266(1): 17-24.

18. FLook EP, Kumar BN. Is there evidence to link acid reflux with chronic sinusitis or any nasal symptoms? A review of the evidence. Rhinology ;49(1): 11-6.

19. Christodoulopoulos P, Cameron L, Durham S, Hamid Q. Molecular pathology of allergic disease. II: Upper airway disease. The Journal of Allergy and Clinical Immunology 2000; 105(2 Pt 1): 211-23.

20. Ponikau JU, Sherris DA, Kephart GM, et al. Features of airway remodeling and eosinophilic inflammation in chronic rhinosinusitis: is the histopathology similar to asthma? The Journal of Allergy and Clinical Immunology 2003; 112(5): 877-82.

21. Rachelefsky GS, Goldberg M, Katz RM, et al. Sinus disease in children with respiratory allergy. The Journal of Allergy and Clinical Immunology 1978; 61(5): 310-4.

22. Strickler A, Lagos M, González B. Deficiencia congénita de complemento: C3 y C4: comunicación de un caso clínico. Rev Chil Enferm Respir 2006; 22(2): 119-25.

23. El-Serag HB, Gilger M, Kuebeler M, Rabeneck L. Extraesophageal associations of gastroesophageal reflux disease in children without neurologic defects. Gastroenterology 2001; 121(6): 1294-9.

24. Phipps CD, Wood WE, GiBson WS, Cochran WJ. Gastroesophageal reflux contributing to chronic sinus disease in children: a prospective analysis. Archives of Otolaryngology-Head \& Neck Surgery 2000; 126(7): 831-6.

25. Muntz HR, Lusk RP. Bacteriology of the ethmoid bullae in children with chronic sinusitis. Archives of Otolaryngology—Head \& Neck Surgery 1991; 117(2): 179-81.

Dirección: Dra. María José Herrera J.

Departamento de Otorrinolaringología, Hospital Barros Luco Trudeau

E mail: ma.jose.hj@gmail.com 